\title{
Evidence for nucleon pair breaking even in the coldest scission configurations of ${ }^{234} U$ and ${ }^{236} U$
}

\author{
C. Signarbieux, 1. Montoya, M. Ribrag, C. Mazur \\ Département de Physique Nucléaire, CEN de Saclay \\ C. Guet, P. Perrin and M. Maurel \\ Département de Recherche Fondamentale, CEN de Grenoble
}

(Reçu le 19 juin 1981, accepté le 13 août 1981)

\begin{abstract}
Résumé. - Les rendements des masses des fragments primaires, mesurés pour les valeurs les plus élevées de l'énergie cinétique libérée dans la fission de ${ }^{233} \mathrm{U}$ et ${ }^{235} \mathrm{U}$ induite par neutrons thermiques, ne révèlent aucune préférence pour les fragmentations en deux noyaux de masse paire. Nos données suggèrent en outre que le mécanisme de brisure de paires interviendrait avant la scission, ce qui revient à dire que la fission à basse énergie serait un processus fortement dissipatif.
\end{abstract}

\begin{abstract}
Primary fragment mass yields, measured at the highest values of the kinetic energy released in the thermal neutron induced fission of ${ }^{233} \mathrm{U}$ and ${ }^{235} \mathrm{U}$, do not reveal any preference for splits into two even-mass nuclei. Moreover our data suggest that the mechanism of pair breaking would take place before scission, namely that the low energy fission would be a strongly dissipative process.
\end{abstract}

A systematic even-odd effect in the fragment charge distribution is a characteristic feature of the thermal neutron-induced fission of ${ }^{233} U$ and ${ }^{235} U$ [1]. The preference for the formation of even- $Z$ fragments has also been observed for any selected value of the kinetic energy of the fragments [2]. These experimental facts have been interpreted as being an indication that the superfluidity of these fissioning systems, known to be completely paired at the saddle point, could be partially preserved until their scission into two fragments [3-6]. In such a hypothesis, the "superfluid" events should be associated with fragments with higher pre-scission kinetic energy and hence with higher final kinetic energy than the " viscous " events. Thus, one should expect that, for the very high kinetic energy values, the splits into two even-even fragments should be strongly preferred if not selected.

This paper proposes to test these predictions in the case of the thermal neutron induced fission of ${ }^{233} \mathrm{U}$ and ${ }^{235} \mathrm{U}$ by measuring primary fragment yields with a resolution able to separate two adjacent masses.

It is known that for these two fissioning systems, the very high kinetic energy fragments are so weakly excited that neither fragment can emit any prompt neutrons [7], so that the primary masses are directly observable. Moreover, we have recently shown that, for this type of fission event, an optimalized time-offlight technique can indeed separate two adjacent mass-ratios fairly well [8]. Briefly, the method is the following : let $m_{i}, v_{i}, e_{i}, l_{i}$ represent respectively the mass, velocity, kinetic energy and flight-path length of the $i$ th fragment $(i=1,2)$. If $\Delta T$ stands for the difference in the time-of-flight of the two fragments, then one obtains :

$$
.1 T=\frac{l_{1}}{v_{1}}-\frac{l_{2}}{v_{2}}
$$

which, using the linear momentum conservation law, becomes :

$$
\Delta T=\frac{l_{1}}{\sqrt{e_{1}}}\left[1-\frac{l_{2}}{l_{1}} \frac{m_{2}}{m_{1}}\right] \sqrt{\frac{m_{1}}{2}} .
$$

It can be noted that, for a fixed $e_{1}$, the values taken by $\Delta T$ constitute a discrete set corresponding to the discrete values of the primary mass ratio. Thus, the time-of-flight spectrum plays the role of a mass spec- 
trometer. As discussed in our previous paper [8], this method fails whenever either of the fragments emits a neutron.

The experimental arrangement is very simple and consists of two $\left(3 \mathrm{~cm}^{2}\right)$ surface barrier detectors placed at 49 and $36 \mathrm{~cm}$ respectively from the centre of a $\left(1 \mathrm{~cm}^{2}\right) \mathrm{U}$ target, the whole being contained in a vacuum chamber. In order to improve the time-offlight resolution, special care was taken to ensure that the detectors and target were strictly parallel so as to minimize flight-length fluctuations. Moreover, the unavoidable velocity-straggling of the fragments inside the target was made as small as possible by the thinness and homogeneity of both the fissile material ( $5 \mu \mathrm{g} \mathrm{cm}^{-2}$ of $\left.\mathrm{UF}_{4}\right)$ and its backing $\left(20 \mu \mathrm{g} \mathrm{cm}^{-2}\right.$ of VYNS). The experiment was performed at the Grenoble High Flux Reactor with an intense thermal neutron beam of $5 \times 10^{9}$ neutrons $\mathrm{cm}^{-2} \mathrm{~s}^{-1}$. For each event detected, the pulse-heights in both detectors and the parameter $\Delta T$ were recorded. An amount of about $3 \times 10^{6}$ and $1.5 \times 10^{6}$ fission events for ${ }^{236} \mathrm{U}$ and ${ }^{234} \mathrm{U}$ respectively have been stored.

The mass-separation capabilities of the lay-out of this experiment are illustrated in figure 1 which shows, for each fissioning system, a raw fragment yield curve

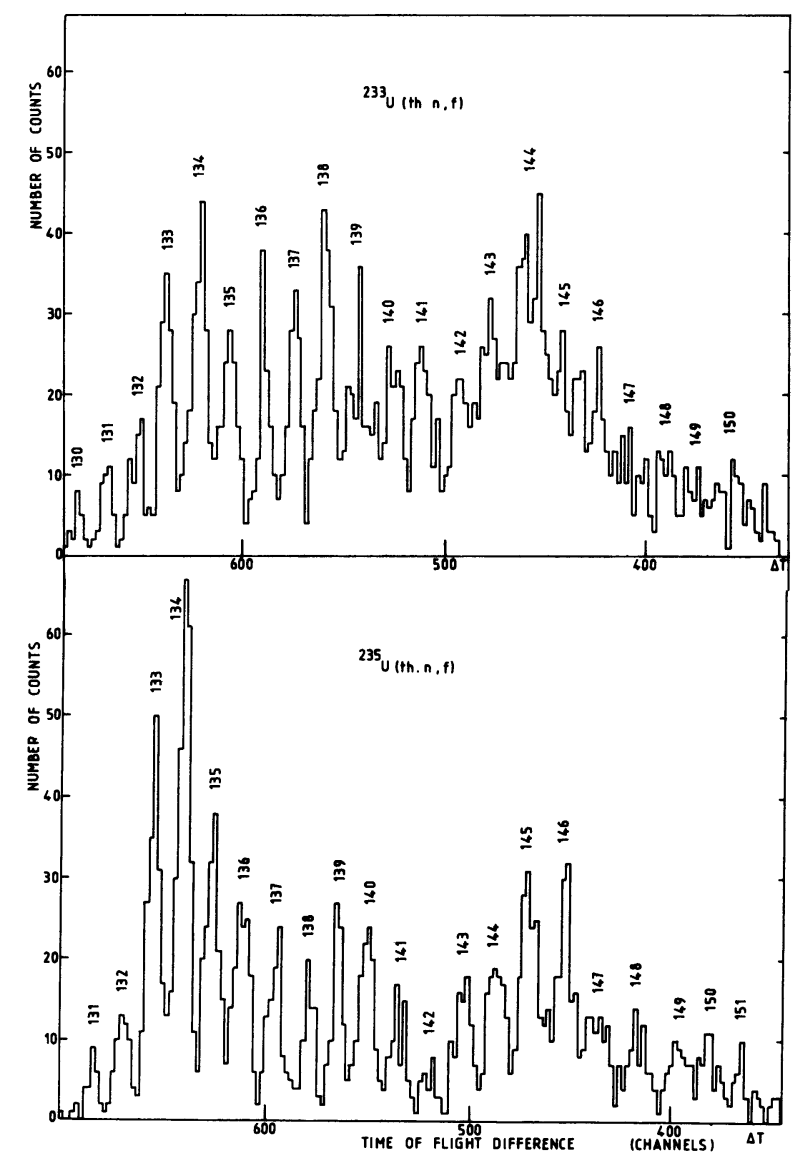

Fig. 1. - Fragment yields plotted against the time-of-flight difference, $\Delta T$, for a $1 \mathrm{MeV}$ window placed at a light fragment kinetic energy of $112 \mathrm{MeV}$. For each peak, the value of the heavy fragment mass is indicated. as a function of $\Delta T$ for a $1 \mathrm{MeV}$ window placed at a light fragment kinetic energy of $112 \mathrm{MeV}$. Each peak has a FWHM of roughly 300 ps while being separated by about 650 ps. The absolute mass-ratio scale, as indicated in figure 1 by the values of the heavy fragment mass, has been established without ambiguity by two independent experimental procedures : the first is the conventional mass calculation from kinetic energies of both fragments [9]; the second consists of identifying the peak at $\Delta T=0$ for which $\frac{m_{2}}{m_{1}}=\frac{l_{1}}{l_{2}}$ (see eq. (1)), by adjusting carefully the $l_{1} / l_{2}$ ratio. It is to note another feature of the experiment : the three parameters $\Delta T, e_{1}, e_{2}$, when associated with the mass and linear momentum conservation relationships, constitute an overdetermined set [10], which allows any false events (due for instance to pile-up in detectors) to be eliminated by means of an ulterior coherence test.

The data analysis consisted in comparing the relative yields of fragmentations into even and odd masses for the highest values of the total kinetic energy release. Such a comparison is not without ambiguity because the yield for a given mass-ratio contains an implicit integration over the charge-ratio distribution and depends also on the available energy of the system at the moment of scission (namely the total minus the deformation energy). In order to overcome the abovementioned difficulties, we determined for each massratio the maximum value of the total kinetic energyTKEMAX-at which the fission yield becomes equal to zero. One would expect that, at such an extreme value, the charge ratio corresponding to the highest possible $Q$-value would be selected and that all the energy of the system would be frozen in deformation [11]. As shown later on, these assumptions are indeed confirmed by our data.

It turned out that the TKEMAX values could be easily determined because the fission yields decrease rapidly with increasing TKE $\left({ }^{1}\right)$ so that the differential uncertainty for two adjacent mass-ratios is less than $0.5 \mathrm{MeV}$. On the other hand, the error on the absolute values, which depends critically on the calibration method used [9], is estimated to be around 2-3 MeV. In figure 2 the TKEMAX values are plotted as a function of the mass-ratio, specified here by the heavy fragment mass. For comparison, the two highest $Q$-values corresponding, for each mass-ratio, to two different charge-ratios are also shown (these $Q$-values have been calculated from the mass table of reference [12]).

As figure 1 shows, fragmentation into odd masses is about as probable as into even masses, but this is clearly demonstrated in figure $2:$ in the full range of mass fragmentation and, although the total excitation

$\left({ }^{1}\right)$ Although this cannot be shown in the present experiment, due to the energy resolution of the detectors used, we feel that the TKEMAX value actually corresponds to a cut-off in the yield (and not to an evanescent tail of the TKE distribution). 


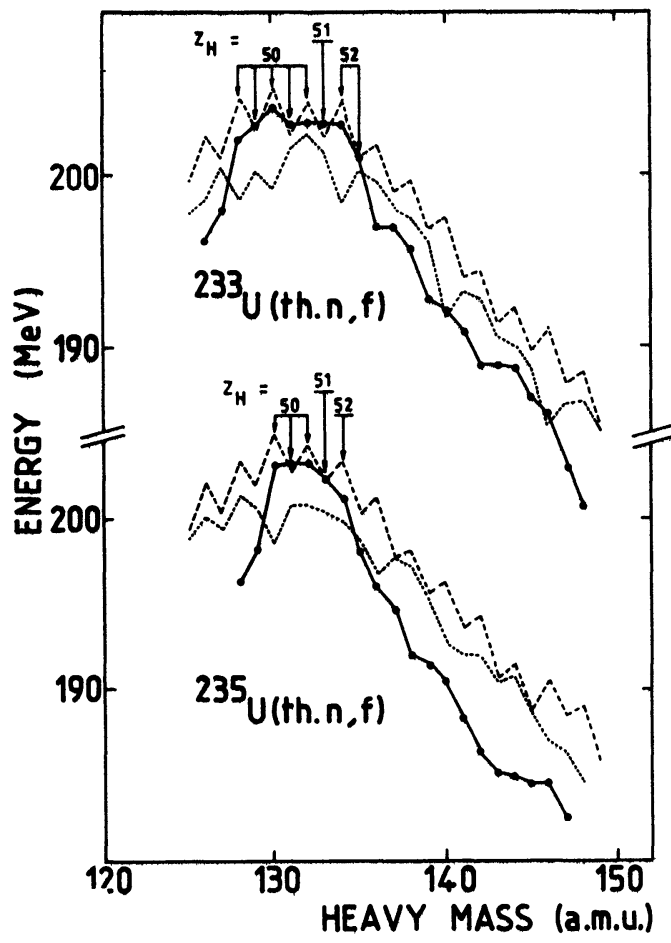

Fig. 2. - Measured maxima of the total kinetic energy of fragments, TKEMAX, are plotted (solid line) against the heavy fragment mass. The dashed line joins the highest possible $Q$-values, whereas the dotted line joins the second highest $Q$-values. In the mass-range where the charge-ratio is identified by energy balance considerations, the value of the heavy fragment charge $Z_{\mathrm{H}}$ is also indicated.

energy of the scission configurations is minimum, the TKEMAX $\left(A_{\mathrm{H}}\right)$ curve is essentially smooth. In other words no preference for the splits into even-mass fragments is observed. In our opinion, this new result permits one to determine the point in the fission process at which pair breaking processes occur. The reasoning is as follows : if the fission dynamics were superfluid, then the pair breaking represented by the experimental observation of odd primary masses should occur at the moment of scission. But the energy required for pair breaking would then show up as a deficit of the order of $2 \mathrm{MeV}$ in TKE, thus creating a residual even-odd effect in the measured TKEMAX $\left(A_{\mathrm{H}}\right)$ values. On the other hand, if the fission dynamics were viscous (pair breakings occurring during the descent towards scission), then at the moment of scission one would observe. at random. splits into two even-mass fragments (each containing both or none of the broken pair nucleons) and into two oddmass fragments (each containing a single nucleon of the broken pair). In this case, no systematic even-odd effect in the TKEMAX $\left(A_{\mathrm{H}}\right)$ would be expected.

Thus, the data of the present experiment suggest that the dynamics of such even-even fissioning systems is essentially viscous, namely that the energy acquired in the collective degree of freedom of fission is strongly dissipated into quasiparticle excitations. Such a breakdown of the pairing correlations during the saddle to scission descent in fission has been predicted by Moretto [13].

Another remarkable observation can be made concerning figure 2 : in a restricted range of massratios, centred around the heavy mass $A_{\mathrm{H}}=132$, the TKEMAX values are so high that they select, for each mass-ratio, only a single charge-ratio (this being the one which makes the $Q$-value maximum). Moreover, the value of the total excitation energy $\left[Q\left(A_{\mathrm{H}}, Z_{\mathrm{H}}\right)-\operatorname{TKEMAX}\left(A_{\mathrm{H}}, Z_{\mathrm{H}}\right)\right]$ for these types of fragmentation is so low that the associated scission configurations are necessarily made of two fragments in, or near, their ground state deformation. But the light fragments associated with the spherical heavy fragments $\left(A_{\mathrm{H}} \approx 132\right)$, are nuclei with 60 to 64 neutrons, which are known to have a prolate deformation in their ground state [14]. It thus follows that the most compact scission configuration for any mode of fragmentation must be thought of as having at least one of its fragments in a prolate deformed state, so that the Coulomb repulsion energy of the fragments does not exceed the $Q$-value. Actually the $\mid Q\left(A_{\mathrm{H}}, Z_{\mathrm{H}}\right)$ - TKEMAX $\left(A_{\mathrm{H}}, Z_{\mathrm{H}}\right) \mid$ energy difference corresponds to the energy required to obtain this necessary minimal deformation.

One is thus led to the conclusion that the relative yields of different fragmentation modes are essentially governed by the available phase space of the system at scission, which is, in turn, determined by the nuclear structure properties of the fragments. We believe that the previously observed experimental facts (even-odd effect in charge distribution, fine structures in mass distribution) are connected to such static properties of the fragments rather than to a certain preservation of the superfluidity of the system during the fission process.

The authors are greatly indebted to J. Van Audenhove for providing exceptionally good uranium targets.

\section{References}

[1] Amiel, S. and Feldsten, H., Phys. Rev. C11 (1975) 845.

[2] Clerc, H. G., lang, W., Wohlfarth, H., Schmitt, K. H. and Schrader, H., in Proc. 3rd Int. Conf. on Nuclei far from Stability, Cargèse 1975 (CERN 75-13, Genf) 1976, p. 509.

[3] Bjornholm, S., Phys. Scr. 10A (1974) 110.
[4] Amiel, S., Feldstein, H. and Izak-Biran, T., Phys. Rev. C15 (1977) 2119.

[5] Nifenecker, H., Blachot, J., Bocquet, J. P., Brissot, R., Crançon, J., Hamelin, C., Mariopoulos, G. and RisTORI, C., Proc. Symp. on Physics and Chemistry of Fission, Jülich 1979 (IAEA, Vienna) 1980, vol. 2, p. 35. 
[6] Clerc, H. G., Lang, W., Wohlfarth, H., Schrader, H. and Schmitr, K. H., Proc. Symp. on Physics and Chemistry of Fission, Jülich 1979 (IAEA, Vienna) 1980, vol. 2, p. 65.

[7] Diven, B. C., Martin, H. C., TAscheK, R. F. and Terrell, J., Phys. Rev. 101 (1956) 3.

[8] Guet, C., Asghar, M., Perrin, P. and Signarbieux, C., Nucl. Instrum. Methods 150 (1978) 189.

[9] Schmitt, H. W., Gibson, W. M., Neiler, J. H., Walter, F. J. and Thompson, T. D., Proc. Symp. on Physics and Che- mistry of Fission, Salzburg 1965 (IEAE, Vienna) 1965 vol. 1, p. 531

[10] SignarbieUx, C., Ribrag, M. and Nifenecker, H., $\mathrm{Nucl}$. Phys. A 99 (1967) 41.

[11] Signarbieux, C., Poitou, J., Ribrag, M. and Matuszek, J., Phys. Lett. 39B (1972) 503.

[12] Garvey, C. T., Gerace, W. J., Jaffe, R. L., Talmi, I. and Kelson, Mr., Rev. Mod. Phys. 414 (1969) part II.

[13] Moretro, L. G., Phys. Lett. 57B (1975) 317.

[14] Ragnarsson, I. and Nilsson, S. G., Phys. Rep. 45 (1978) 1. 